EPJ Web of Conferences 59, 03006 (2013)

DOI: $10.1051 /$ epjconf/20135903006

(C) Owned by the authors, published by EDP Sciences, 2013

\title{
High-resolution X-ray imaging in fast ignition experiment using Gekko and LFEX lasers
}

\author{
M. Koga ${ }^{\text {, }}$ Y. Ishii, T. Sogo, K. Shigemori, H. Shiraga, S. Fujioka, H. Azechi \\ and FG-02 Experimental Campaign Team
}

Institute of Laser Engineering, Osaka University, 2-6 Yamada-Oka, Suita, Osaka 565-0871, Japan

\begin{abstract}
We improved diagnostic instruments to measure X-ray images in a hard X-ray harsh environment and succeeded in obtaining clear images with $\mathrm{X}$-ray framing camera and $\mathrm{X}$-ray streak camera in fast ignition experiment conducted in 2011 (FG-02 Experimental Campaign). We found that high-energy X-ray signals could be used as an indicator of the LFEX laser injection time relative to the imploded core. The LFEX laser injection time was estimated with better than $10 \mathrm{ps}$ accuracy. Time-resolved 2D X-ray images suggested that shapes and motions of imploded core plasmas were improved by changing the configuration of the implosion lasers.
\end{abstract}

\section{INTRODUCTION}

Fast ignition [1-4] is one of the methods of achieving laser fusion. This scheme separates lasers into two systems: one for implosion and the other for heating the imploded fuel core. Fast ignition is considered as a powerful method because this scheme can achieve fusion gain with about 10 times smaller laser energy than the central ignition scheme. However, for effective heating, it is still needed to know the implosion hydrodynamics and the heating mechanism of the imploded core plasma. The size of the imploded core plasma becomes less than 100 micron and its heating process lasts only less than $100 \mathrm{ps}$. Therefore, high spatial and temporal resolution measurements are necessary. We have used X-ray framing cameras (XFC) and X-ray streak cameras (XSC) for this purpose. The temporal and spatial resolutions of XFC and XSC are described in section 2. In the fast ignition experiment, hard X-rays above $100 \mathrm{keV}$ were generated from hot electrons through bremsstrahlung [5]. These X-rays caused many troubles, such as malfunction of control devices, intense signal overlaps (See Fig. 1(a)) and cathode discharges (See Fig. 2(a)). In this paper, we report our improvements to overcome such a harsh environment and obtained results.

\section{EXPERIMENTAL SETUP}

The experiments were performed using the Gekko XII and the LFEX laser system [6] at the Institute of Laser Engineering, Osaka University. The wavelength of the GXII laser was 0.53 micron and the pulse duration was $1.5 \mathrm{~ns}$. The total energy of the GXII implosion laser was about $2 \mathrm{~kJ}$. The wavelength of the LFEX laser is 1.05 micron and the pulse duration was set to $1 \sim 5 \mathrm{ps}$. The targets were deuterated plastic shells with gold cones. The diameter of the shells was 500 microns. The distance between the cone tip and the center of its shell was 50 microns.

\footnotetext{
ae-mail: koga-m@ile.osaka-u.ac.jp
}

This is an Open Access article distributed under the terms of the Creative Commons Attribution License 2.0, which permits unrestricted use, distribution, and reproduction in any medium, provided the original work is properly cited. 

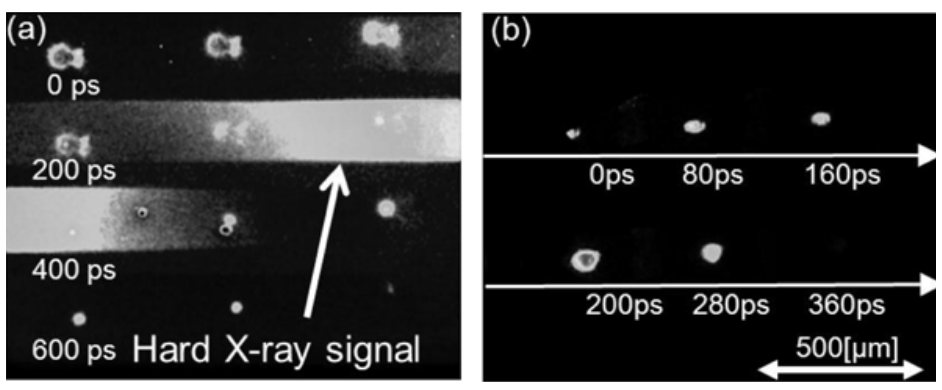

Figure 1. XFC images obtained (a) with the pinhole system only, (b) with the pinhole system and X-ray mirrors. GXII laser energy was (a) $280 \mathrm{~J} /$ beam and (b) $275 \mathrm{~J} /$ beam. LFEX laser energy and the pulse width were (a) $210 \mathrm{~J}$ and $5 \mathrm{ps}$, and (b) $408 \mathrm{~J}$ and $1.5 \mathrm{ps}$, respectively.

X-ray framing camera (XFC) was used to obtain the time-resolved 2D X-ray images directly [7-9]. Details of our XFC system are described in Ref. [9]. An array of $10 \mu \mathrm{m}$ diameter pinholes was used to create separate 2D X-ray images. A blast shield made of $25-\mu \mathrm{m}$-thick beryllium was attached in front of the pinhole disk in order to shield debris and soft X-rays. The images were projected on a multi channel plate (MCP) with a size of $40 \mathrm{~mm} \times 50 \mathrm{~mm}$. The MCP had gold striplines across its face. An electric pulse was sent down a stripline to obtain the image under each pinhole only when the pulse was present at that location on the MCP. The intensified images were recorded with a CCD camera. X-ray images were magnified 20 times on CCD. The spatial resolution of XFC became 17.3 microns. The time interval between frames was defined by the velocity of the electric pulses and it was set about $80 \mathrm{ps}$. This time resolution was high enough to observe the implosion process, but it was slightly too low to observe the heating process.

To complement the time resolution of XFC, a multi-imaging X-ray streak camera (MIXS) was used in our experiment. Details of MIXS are described in Ref. [10]. The tilted multi pinhole array was used to obtain streaked images of different parts of the core plasma. 2D X-ray images were reconstructed from a set of 1D images. The spatial resolution and the temporal resolution of MIXS were 22.4 micron and $10.5 \mathrm{ps}$, respectively. The time resolution was high enough to observe the heating process.

\section{RESULTS AND DISCUSSION}

\subsection{Measurements in a harsh environment}

Figure 1(a) shows the XFC image obtained with the pinhole system only. GXII laser energy was $280 \mathrm{~J} /$ beam. LFEX laser energy was $210 \mathrm{~J}$ with $5 \mathrm{ps}$ pulse width. Hard X-rays generated from hot electrons penetrated the substrate of the pinhole array and generated electrons throughout the MCP. They caused bright signals on the XFC image. As shown in Fig. 1(a), this non-imaged hard X-ray signal overlaps 2D X-ray images of the imploded core plasma and hides them. To clear this problem, we introduced X-ray mirrors made of platinum to our XFC. Only thermal X-rays $(<5 \mathrm{keV})$ were reflected by these mirrors. The direct incidence of hard X-rays through the pinhole disk was shielded by a lead block. As shown in Fig. 1(b), a clear thermal X-ray image was obtained after this improvement although LFEX laser energy was higher (408 J and 1.5 ps) than in Fig. 1(a).

Figure 2(a) shows the streaked MIXS image obtained with the slit system only. Gekko XII laser energy was $322 \mathrm{~J} /$ beam. LFEX laser energy was $548 \mathrm{~J}$ with 5 ps pulse width. Bright spots were cathode discharges caused by hard X-rays. These discharges not only hid streaked images but also damaged the cathode. In order to suppress the direct incidence of hard X-rays, a thick tungsten shield with a slit was used in front of the photocathode. Figure 2 (b) shows the streaked MIXS image obtained with the slit system and shields. Gekko XII laser energy was $348 \mathrm{~J} /$ beam. LFEX laser energy was $517 \mathrm{~J}$ with $1.5 \mathrm{ps}$ 

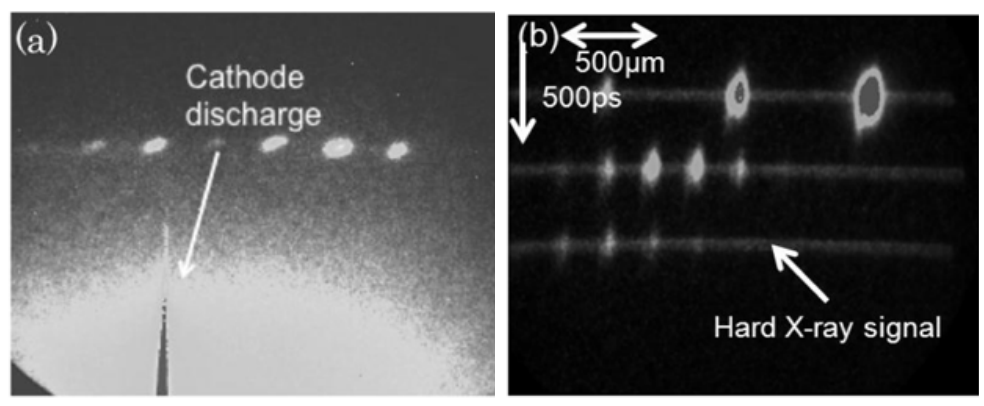

Figure 2. MIXS images obtained (a) with the slit system only, (b) with the slit system and shields. (a) Gekko XII laser energy was $322 \mathrm{~J} /$ beam. LFEX laser energy was $548 \mathrm{~J}$ with 5 ps pulse width, (b) Gekko XII laser energy was $348 \mathrm{~J} /$ beam. LFEX laser energy was $517 \mathrm{~J}$ with 1.5 ps pulse width.

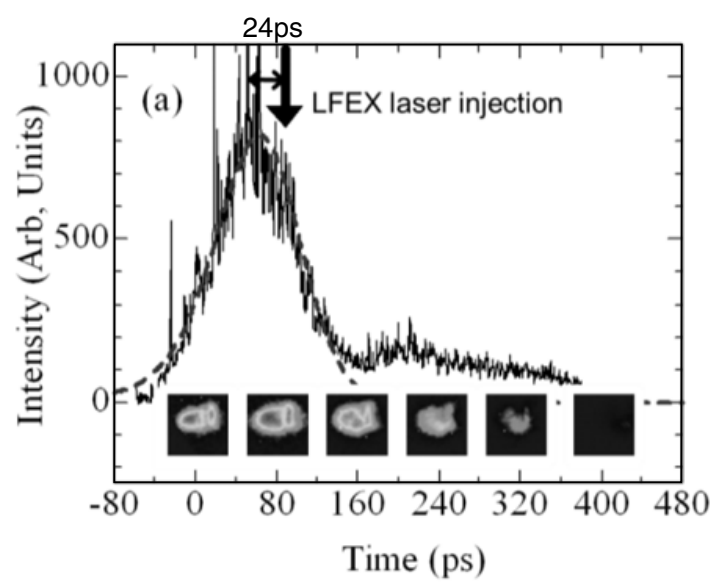

Figure 3. Temporal evolution of the hard X-ray intensity obtained from the XFC data. A dotted line shows the calculated hard X-ray signal. Corresponding 2-dimensional X-ray images are shown in the same figure.

pulse width. In Fig. 2(b), it is clearly seen that cathode discharges do not occur. Although hard X-ray signals through the slit remained, we can subtract these signals from streaked images easily because these signals are distributed to all slit area homogeneously.

\subsection{LFEX laser injection timing}

The injection time of a heating laser to the imploded core plasma is very important for effective neutron generation [4]. The generation of hot electrons and hard X-rays are considered to be very fast processes equivalent to the pulse duration of the LFEX laser. Therefore, we can use hard X-ray signals as an indicator of the injection time of the LFEX laser [5]. We removed a part of the lead shield from our XFC and obtained a small hard X-ray signal. The LFEX injection time is estimated by using a model calculation. Details of this calculation will be described in a forthcoming paper.

Figure 3 shows the temporal evolution of the hard X-ray intensity obtained from the XFC data. A dotted line shows the calculated hard X-ray signal. It is seen that the calculated gain profile agrees well with the measured hard X-ray temporal profile. Corresponding 2D X-ray images are shown in the same figure. These images show side views of the target. In each image, the round imploded core is seen at 


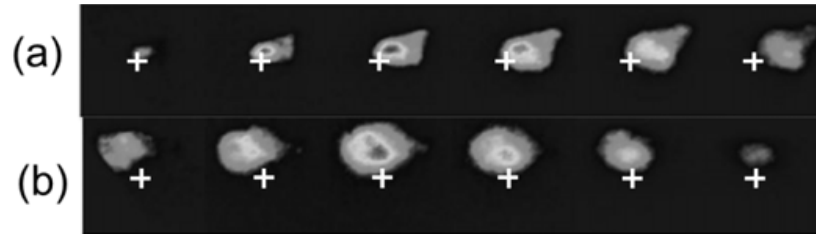

Figure 4. XFC images of the imploded core plasma obtained in (a) 9-beam implosions and (b) 12-beam implosions. Time resolved images are aligned from left to right. White crosses show the chamber center.

the center of the frame and an emission of the cone tip is seen on the right side of it. In Fig. 3, the imploded core plasma becomes brightest in the 2 nd frame and then vanishes gradually. The maximum compression of the imploded core is considered to occur around this frame. The peak position of hard $\mathrm{X}$-ray intensity is seen at $\mathrm{t}=60 \mathrm{ps}$ in horizontal time axis. From calculation, it was found that the LFEX injection time was $24 \mathrm{ps}$ after the peak time of the hard X-ray signal. Therefore, the LFEX injection time should be at $\mathrm{t}=84 \mathrm{ps}$ in this case. The error bar to estimate the injection time is less than $10 \mathrm{ps}$ including the fitting error of the calculated hard X-ray signal.

As shown in Fig. 2(b), hard X-ray signals are also obtained in the MIXS images. We also estimated LFEX injection times from these signals. It was found that LFEX injection times estimated from the XFC data and the MIXS data were consistent. This means that this method to estimate the heating laser injection time is reliable.

\subsection{Control of the imploded core plasma using 12 laser beams}

Usually, three laser beams near the cone are not used for implosion because they irradiate the cone. However, it was found that this laser imbalance caused a non-uniform implosion and made the imploded core plasma move toward the cone. The imploded core plasma may collide with the cone tip and break it. This may become a serious problem by disturbing the effective generation of hot electrons from the cone tip. Therefore, we proposed a new concept of implosion, which we called "12-beam implosions". In 12-beam implosions experiments, three laser beams near the cone were shifted so as not to irradiate the cone. Longer cones $(2.7 \mathrm{~mm})$ compared with the usual cones $(1 \mathrm{~mm})$ were used to prevent the irradiation with residual fundamental frequency of three laser beams inside a cone.

Figure 4(a) and 4(b) show XFC images of imploded core plasmas obtained in the usual 9-beam implosions and 12-beam implosions, respectively. The position of the chamber center was defined by $\mathrm{X}$-ray images of Au cross target shots. Au cross target was made with Au coated carbon fibers ( 7 micron diam. and 500 micron length) and set at the chamber center. It is indicated with a white cross. It is clearly seen that the imploded core plasma shows a more spherical form in 12-beam implosions than that in 9-beam implosions. In 9-beam implosions, imploded core plasmas are generated near the cone tip and move toward the cone. On the other hand, in 12-beam implosions, imploded core plasmas are generated away from the cone tip and move toward the cone slowly. It was found that the velocity of imploded core plasmas in 12-beam implosions was reduced to about half of that in 9-beam implosions. The details of the imploded core plasma generated in 12-beam implosions experiment, such as the density and the temperature, will be measured in the next experiment.

\section{References}

[1] N. G. Basov, S. Yu. Gus'kov, and L. P. Feoktistov, J. Soviet Laser Research 13, 396 (1992)

[2] M. Tabak, J. Hammer, M. E. Glinsky, W. L. Kruer et al., Phys. Plasmas 1, 1626 (1994)

[3] R. Kodama, P. A. Norreys, K. Mima, A. E. Dangor et al., Nature 412, 798 (2001) 


\section{IFSA 2011}

[4] R. Kodama, H. Shiraga, K. Shigemori, Y. Toyama et al., Nature 418, 933 (2002)

[5] M. Koga, K. Shigemori, H. Shiraga, H. Azechi, J. Phys. Conf. Series 244, 022043 (2010)

[6] N. Miyanaga, H. Azechi, K.A. Tanaka, T. Kanabe et al., J. de Physique IV 133, 81 (2006)

[7] D. K. Bradley, P. M. Bell, J. D. Kilkenny, R. Hanks et al., Rev. Sci. Instrum. 63, 4813 (1992)

[8] J. D. Kilkenny, Rev. Sci. Instrum. 63, 4688 (1992)

[9] M. Koga, T. Fujiwara, T. Sakaiya, M. Lee et al., Rev. Sci. Instrum. 79, 10E909 (2008)

[10] H. Shiraga, M. Heya, A. Fujishima, O. Maegawa et al., Rev. Sci. Instrum. 66, 722 (1995) 